Supporting Information

\title{
Supported Metal Clusters: Fabrication and Application in Heterogeneous Catalysis
}

Chunyang Dong ${ }^{1}$, Yinlong Li $^{2}$, Danyang Cheng ${ }^{1}$, Mengtao Zhang ${ }^{1}$, Jinjia Liu ${ }^{3,4}$, Yang-Gang Wang ${ }^{2}$,

Dequan $\mathrm{Xiao}^{5}$, and Ding $\mathrm{Ma}^{1 *}$

1. Beijing National Laboratory for Molecular Sciences, College of Chemistry and Molecular Engineering and College of Engineering, and BIC-ESAT, Peking University, Beijing 100871, China

2. Department of Chemistry and Guangdong Provincial Key Laboratory of Catalysis, Southern University of Science and Technology, Shenzhen 518055, Guangdong, China

3. State Key Laboratory of Coal Conversion, Institute of Coal Chemistry, Chinese Academy of Sciences, Taiyuan, Shanxi 030001, China

4. National Energy Center for Coal to Liquids, Synfuels China Technology Co., Ltd, Beijing 101400, China

5. Center for Integrative Materials Discovery, Department of Chemistry and Chemical Engineering, University of New Haven, CT 06516, USA

*Corresponding author: dma@pku.edu.cn 
Table S1. Correlation between atom numbers and average metal-metal coordination numbers (M-M C.N.) over a series of face-centered cubic (fcc) cuboctahedrons.

\begin{tabular}{cc} 
Atom numbers & Average M-M C.N. \\
\hline $1^{\mathrm{a}}$ & 0 \\
$3^{\mathrm{a}}$ & 2 \\
$13^{\mathrm{a}}$ & 4.9 \\
$50^{\mathrm{a}}$ & 7.4 \\
147 & 9.2 \\
309 & 9.8 \\
561 & 10.3 \\
923 & 10.5 \\
$1034^{\mathrm{a}}$ & 10.4 \\
1415 & 10.7 \\
2057 & 10.9 \\
2869 & 11 \\
3871 & 11.1 \\
5083 & 11.2 \\
6525 & 11.2 \\
\hline
\end{tabular}

a the atom numbers and corresponding average M-M C.N. are based on a series of supported fcc cuboctahedrons (in consistent with the model showed in Scheme 1a), while others in this table are based on the free-standing fcc cuboctahedrons. The minor difference of the average M-M C.N. between the free-standing and supported fcc cuboctahedrons for larger metal NPs $(N>100)$ is neglected in the plotting of Scheme 1c. 